\title{
Size does matter: model selection of shark attack case
}

\section{Andri Wibowo*}

Keywords: AIC, model, shark, size, unprovoked

*U. o. Indonesia, West Java, 16424. Indonesia. Correspondence email: paleobio2020@gmail.com

\begin{abstract}
Shark unprovoked attacks consist of fatal and non-fatal cases Numerous cases have been reported involving shark species from Carcharhinus melanopterus with length of $145.5 \mathrm{~cm}$ to half-ton Carcharodon carcharias. Currently there are more $(\mathrm{P}<0.05)$ unprovoked non-fatal cases with the average is 28.46 cases/shark species (95\%CI: 3.86-53.1) than unprovoked fatal cases, which the average is 5.12 cases /shark species (95\%CI: -0.075-10.3). Hence this paper seeks to select the best shark size model that correlates with the unprovoked fatal and non-fatal cases. The studied sharks consist of 24 shark species with the average length is $268.18 \mathrm{~cm}(95 \% \mathrm{CI}: 230-306 \mathrm{~cm})$ and the average weight is $225.42 \mathrm{~kg}$ (95\% CI: $128-323 \mathrm{~kg}$ ). Based on the model and as described by low values of AIC and the highest values of $\mathrm{R}^{2}$ and adjusted $\mathrm{R}^{2}$, shark weight followed by combinations of shark weight and length produced unprovoked fatal and non-fatal cases best models. The model for explaining unprovoked fatal cases is the shark weight with high numbers of cases observed in large size shark (weight $\sim$ fatal cases, AIC $=165.359, R^{2}=0.72$, Adj. $R=0.71$ ). While for non-fatal cases, the best model is also the shark weight (weight $\sim$ non fatal cases, AIC = 246.93. $\mathrm{R}^{2}=0.63$. Adi. $\mathrm{R}=0.59$ ).
\end{abstract}

\section{Introduction}

Sharks have been an interest to humans and scientific study because they represent one of only a few groups of marine animals that negatively interact with human on a regular basis. This uncommon interaction commonly referred to as unprovoked shark attack. An unprovoked attack is defined as a bite or near bite (fended off by human intervention) of a person (or the board on which he/she is perched) in the shark's natural habitat without presence of any human provocation. ISAF (2016) has reported 75-100 unprovoked attacks happen annually and most of the attacks result in minor injuries akin to that of a dog bite, yet 6 of those unprovoked attacks result in fatal cases .

Over the past three decades, the number of unprovoked shark bites on humans has increased globally. This increasing trend has been documented in multiple countries. Currently, the unprovoked cases are theoretically related to increasing recreational activities mainly surfing (Woolgar et al. 2001, Neff 2012, Ferretti 2015). Currently, growing populations and related tourisms in beaches are assumed to be the 
determinant factors of unprovoked attack cases. An increased number of attacks likely does not relate to the increase in shark attack frequencies alone. Whereas, human populations are growing and this has led to the increase in numbers of people swimming, surfing, and diving in the ocean.

Most studies on detrimental factors underlying shark attacks are emphasizing on environmental and anthropogenic factors including the human activities. Whereas, information on how the shark biological factors contribute to the unprovoked cases is still limited. This information is very important considering that there are 500 species of sharks swimming in the world's ocean sizing from the length of a human hand to more than 12 meter long. In fact, half of all shark species are less than one meter long. Then the question addressed in this research is does the shark size contributes to the unprovoked cases?

\section{Methods}

The data of unprovoked shark attack cases for 24 species were compiled from International Shark Attack File (ISAF). The unprovoked case data consists of non-fatal and fatal cases. Those case data then combined with the data of shark body size including length and size obtained through literature surveys.

Analysis data consists of correlation analysis, Principal Component Analysis, and Akaike (AIC) model selection. To build the model, 2 explanatory variables including shark length and weight with combinations of those variables were included in the analysis to develop the model.

\section{Results}

The frequencies of non-fatal cases are higher than fatal cases and almost 4 folds $(\mathrm{P}<0.05)$ (Figure 1$)$. Compiling data from 24 species, the average of non-fatal cases is 28.46 cases/shark species (95\% CI: 3.8653.1) while the average of fatal cases is 5.12 cases /shark species (95\%CI: -0.075-10.3).

There is positive correlation between length and weight of 24 shark species (Figure 2). Those consist of 24 shark species with the average length is $268.18 \mathrm{~cm}$ (95\% CI: $230-306 \mathrm{~cm}$ ) and the average weight is $225.42 \mathrm{~kg}$ (95\%CI: 128-323 kg). The Carcharhinus genus is dominating the medium and small size sharks. While great white shark is prominent for its length and weight. The size of shark is also correspondent to non-fatal and fatal cases. Both cases are correlated with the either length or weight of sharks (Figure 3). Based on PCA, there are 3 species that correlate more to the unprovoked cases. Those species are commonly the shark that has large body size. 12 Carcharhinus genus studied in here with average length is $241.70 \mathrm{~cm}$ (95\%CI: $208-275 \mathrm{~cm})$ and weight is $168.50 \mathrm{~kg}$ (95\%CI: 98-239 kg) are correlated with the 17.5 non-fatal cases/Carcharhinus species (95\% CI: 2.9-32.1) and 2.6 fatal cases/ Carcharhinus species (95\%CI:-1.35-6.67).

Based on the model (Table 1) and as described by low values of AIC and the highest values of $\mathrm{R}^{2}$ and adjusted $\mathrm{R}^{2}$, shark weight followed by combinations of shark weight and length produced unprovoked fatal and non-fatal cases best models. The model for explaining unprovoked fatal cases is the shark weight with high numbers of cases observed in large size shark (weight $\sim$ fatal cases, $A I C=165.359, \mathrm{R}^{2}=$ 
0.72 , Adj. $\mathrm{R}=0.71$ ). While for non-fatal cases, the best model is also the shark weight (weight $\sim$ non fatal cases, $\mathrm{AIC}=246.93, \mathrm{R} 2=0.63$, Adj. $\mathrm{R}=0.59$ ).

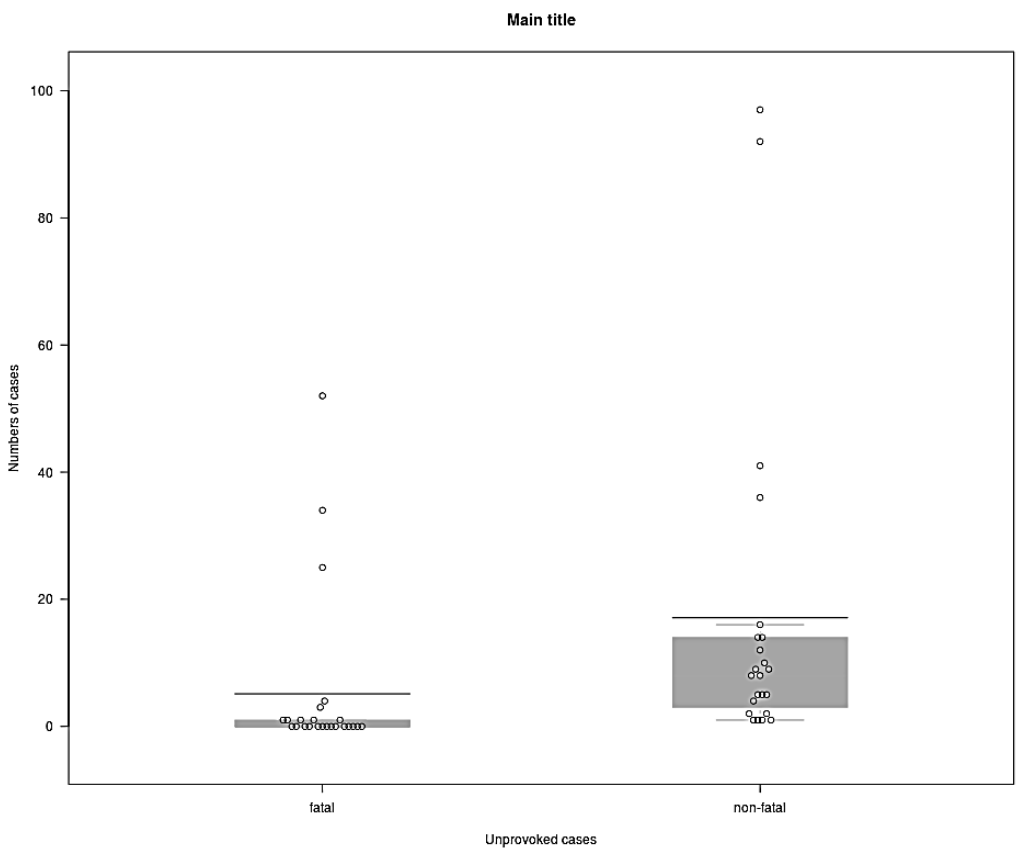

Figure 1. A comparison of fatal (left) and non-fatal (right) provoked cases of 24 shark species.

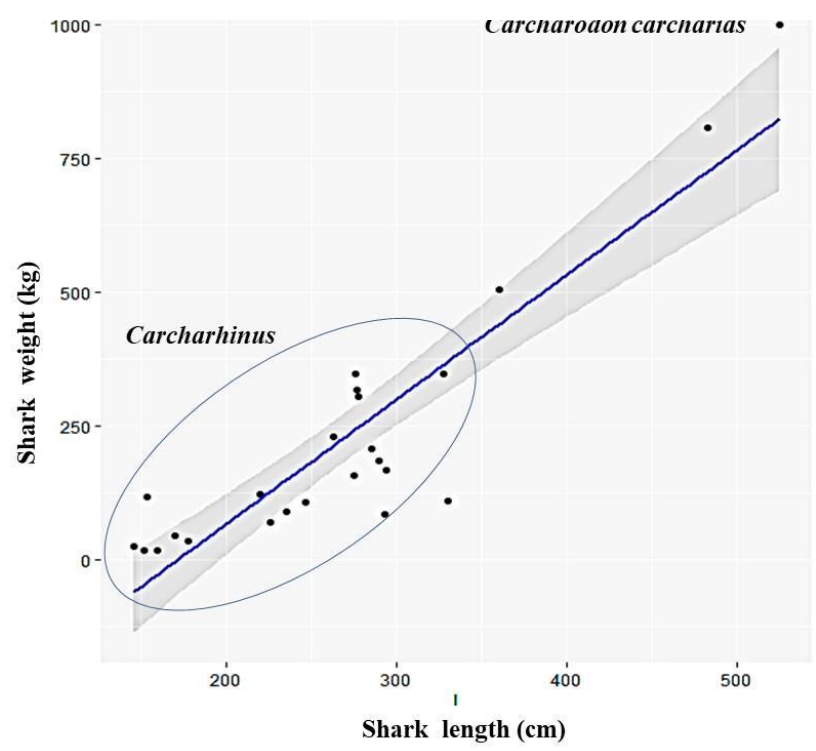

Figure 2. Correlations of shark length (cm) (x axis) with shark weight (kg) (y axis). 

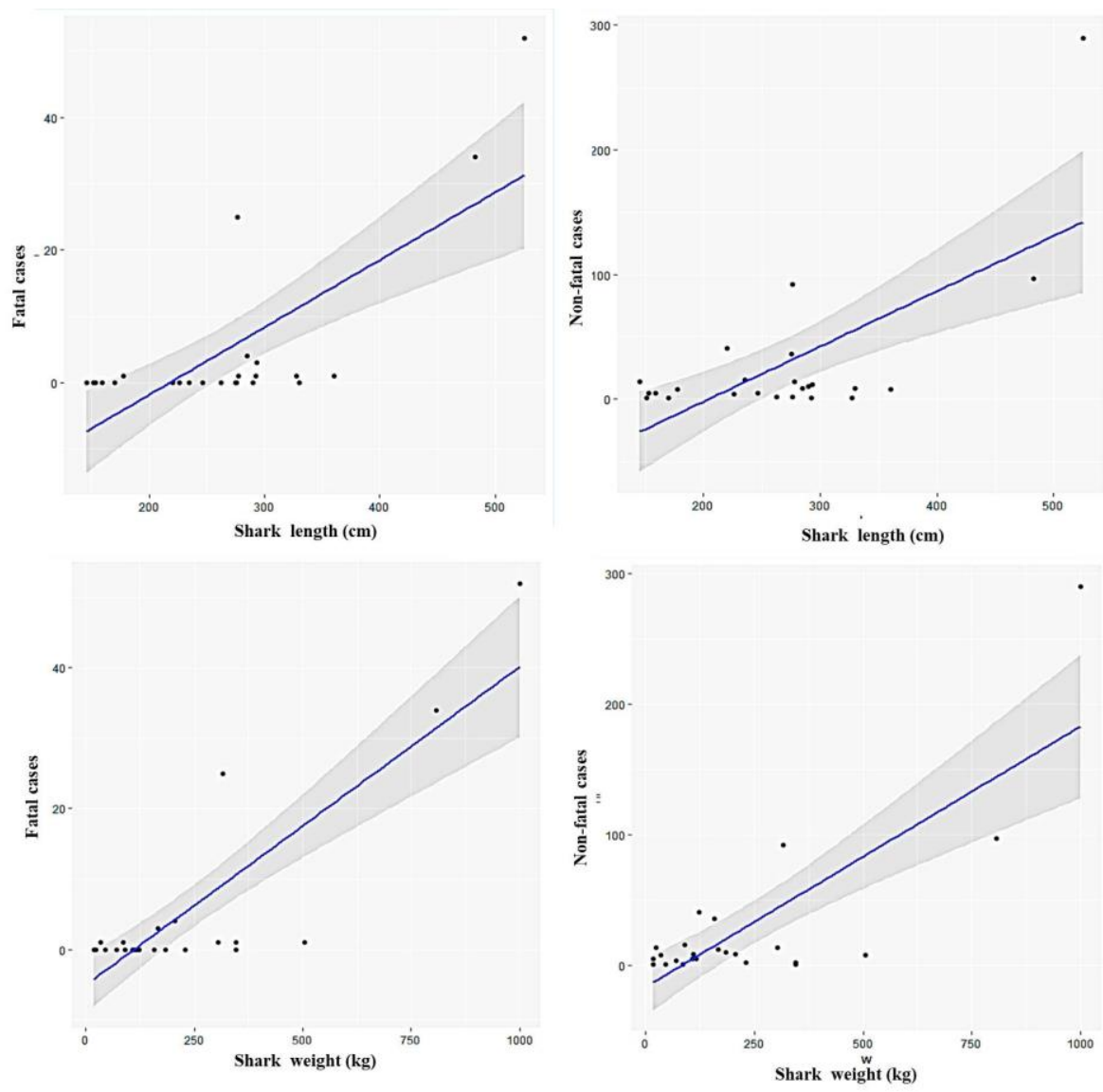

Figure 3. Correlations of shark length $(\mathrm{cm})$ and shark weight $(\mathrm{kg})$ (x axis) with numbers of fatal and non fatal cases (y axis).

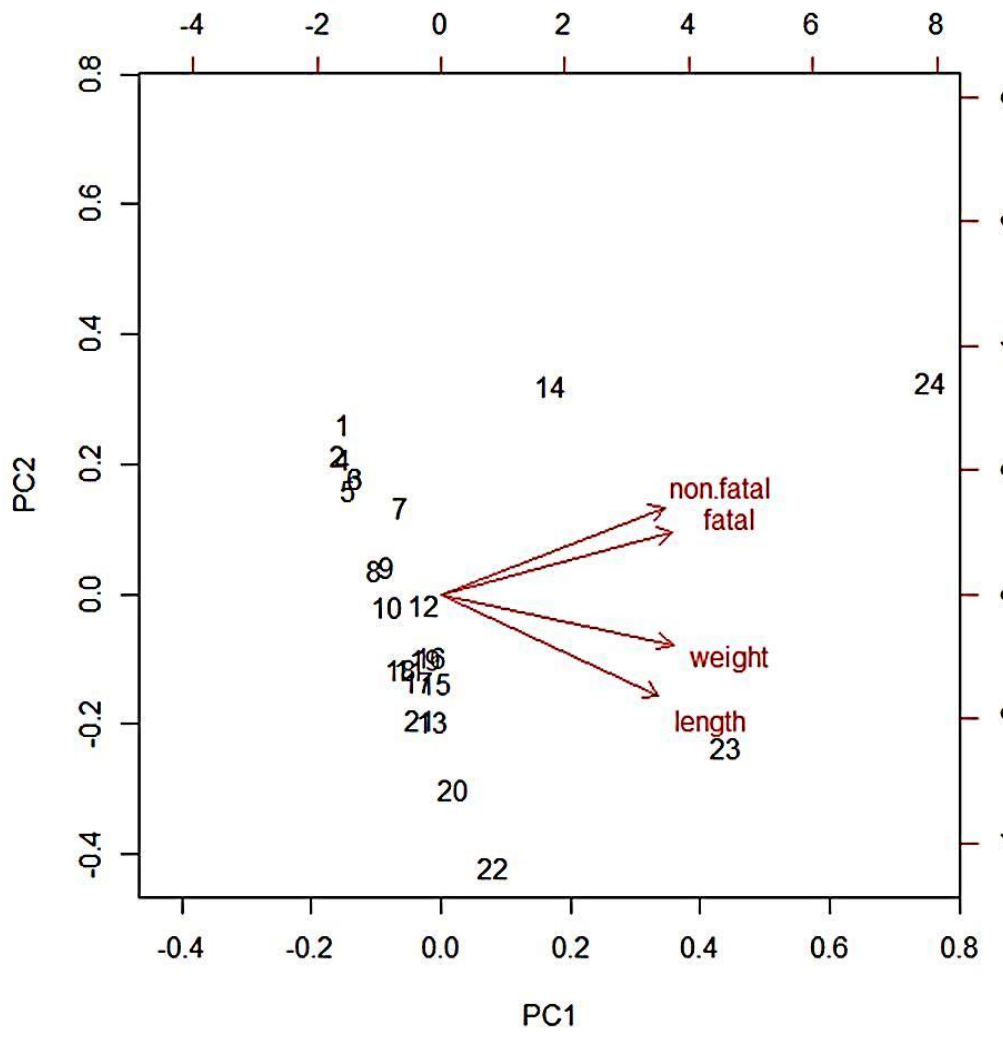

Species name:

1.Carcharhinus melanopterus

2. Triakis semifasciata

3. Carcharhinus plumbeus

4. Triaenodon obesus

6. Galeorhinus galeus

6. Carcharhinus amblyrhynchos

7. Carcharhinus limbatus

8. Carcharhinus perezi

9. Carcharhinus brevipinna

10. Notorynchus cepedianus

11. Lamna nasus

12. Carcharias taurus

13. Carcharhinus falciformis

14. Carcharhinus leucas

15. Carcharhinus brachyurus

16. Prionace glauca

17. Negaprion brevirostris

18. Carcharhinus galapagensis

19. Carcharhinus longimanus

20. Carcharhinus obscurus

21. Ginglymostoma cirratum

22. Isurus oxyrinchus

23. Galeocerdo cuvier

24. Carcharodon carcharias

Figure 4 Principal Component Analysis for shark size factors (weight, length) and unprovoked cases (fatal, non-fatal) among 24 shark species. 
Tabel 1. Model of unprovoked cases (fatal, non-fatal) with shark size factors (weight, length)

\begin{tabular}{lrllll}
\hline \multicolumn{1}{c}{ Model } & AIC & \multicolumn{1}{c}{$\mathrm{R}^{2}$} & \multicolumn{1}{c}{ Adj R $^{2}$} & \multicolumn{1}{c}{ Residual } & P \\
\hline (fatal) $\sim$ (length) & 176.917 & 0.552 & 0.5317 & 8.894 & 0.00 \\
(fatal) $\sim$ (weight) & 165.359 & 0.7232 & 0.7107 & 6.991 & 0.00 \\
(fatal) $\sim($ weight $)($ length $)$ & 167.054 & 0.7267, & 0.7007 & 7.11 & 0.00 \\
(non fatal) $\sim($ length $)$ & 255.549 & 0.4691 & 0.445 & 45.76 & 0.00 \\
(non fatal) $\sim($ (weight $)$ & 246.935 & 0.6292 & 0.6124 & 38.25 & 0.00 \\
(non fatal) $\sim$ (weight)(length) & 248.578 & 0.6347, & 0.5999 & 38.86 & 0.00 \\
\hline
\end{tabular}

\section{Discussion}

Study on causative factors of unprovoked shark attack cases is growing recently (Lagabrielle et al. 2018, Midway et al. 2019) Unprovoked shark bite incidence is increasing world-wide. It becomes more apparent in shark attack hotspots range from the United States, South Africa, Australia, Brazil, Reunion Island and to Bahamas. Blake et al. (2016) have confirmed the increases in shark bite incidence are likely correlated to rises in human population combined with other anthropogenic related causative factors, including habitat destruction/modification, water quality degradation, climate change, and anomalous weather. Those combined factors have disrupted the natural balance of an area at a local or regional level and increases the probability of shark-human encounter lead to unprovoked cases. Whereas, the increase in unprovoked shark bite could not be explained entirely by increases in human population (Mcphee 2014). This study presents that the unprovoked cases related to predator and prey mainly the body size relationship can be another underlying causes.

In this study and based on PCA analysis, there are 3 shark species that significantly correlate with the unprovoked cases and this excludes the small and medium size sharks mainly from Carcharhinus genus group. Actually, there are 3 shark species that attract much of the attention regarding shark attack cases. Those species are the bull shark (Carcharhinus leucas), tiger shark (Galeocerdo cuvier), and white shark (Carcharodon carcharias). These species are of interest to science because they are large charismatic megafauna status and for their association with reports of fatal shark attacks.

The model shows that larger size shark is correlated more with unprovoked case frequencies compared to small and medium size sharks. This correlation is corroborated with the predator and prey relationship where body size is known to play a critical role in determining patterns of prey selection (Osenberg \& Mittelbach GG. 1989, Nakazawa et al. 2013). Unprovoked shark attack cases in this study are representing the cases involving human as the selected shark prey. Considering the average of human height is $160.3 \mathrm{~cm}$ globally (Bentham et al. 2016) and this may explain the case trend as function of 
predator-prey body size relationships. For some shark species of Carcharhinus genus including $C$. melanopterus, C. plumbeus, C. galeus, C amblyrhynchos, and Triakis semifasciata, Triaenodon obesus, they may avoid to select human with size almost the same of its body length and this can be seen in the lower unprovoked cases compared to other large size sharks. While, when the length of shark increase as can be seen in medium size and large great white shark, human will be preyed and this increases significantly the unprovoked cases.

The study is not presented the direct predator-prey correlation of shark size with human size, whereas it explains that large shark prefers more to prey large size prey including human and this is represented in the high frequency of unprovoked cases. While the low unprovoked cases in small shark because the size of human sizing $160 \mathrm{~cm}$ is to large from small sharks. The general patterns of prey size use by shark in this study correspond well with previous studies of predation by fishes (Scharf et al. 2000). In fish, prey size is a linear function of predator length.

\section{Conclusions}

The shark attack case is interesting and it is a growing study. It becomes imminent since the attacks are the functions of numerous factors. To conclude, this paper has provided empirical evidences with robust analysis how shark size correlates to unprovoked cases.

\section{References}

Bentham J, Di Cesare M, Stevens G, Zhou B. et al. 2016. A century of trends in adult human height. eLife Sciences. 5.

Blake K. Chapman, Daryl McPhee 2016. Global shark attack hotspots: Identifying underlying factors behind increased unprovoked shark bite incidence Ocean \& Coastal Management. 133:72-84

Ferretti F, Jorgensen S, Chapple TK, De Leo G, Micheli F. 2015.Reconciling predator conservation with public safety. Front. Ecol. Environ.;13:412-417.

Gaeta JW, Ahrenstorff TD, Diana JS, Fetzer WW, Jones TS, Lawson ZJ, et al. 2018. Go big or ... don't? A field-based diet evaluation of freshwater piscivore and prey fish size relationships. PLoS ONE 13(3):

ISAF.2016. International Shark Attack File

Lagabrielle E, Allibert A, Kiszka JJ, Loiseau N, Kilfoil JP, Lemahieu A. 2018. Environmental and anthropogenic factors affecting the increasing occurrence of shark-human interactions around a fast-developing Indian Ocean island. Scientific reports, 8(1), 3676.

Mcphee D. 2014. Unprovoked Shark Bites: Are They Becoming More Prevalent?. Coastal Management. 42. 478-492.

Midway SR, Wagner T, Burgess GH .2019. Trends in global shark attacks. PloS one, 14(2), 
Nakazawa T, Shin-ya O, Masayuki U. 2013 Predator-prey body size relationships when predators can consume prey larger than themselves Biol. Lett

Neff C. 2012. Australian beach safety and the politics of shark attacks. Coast. Manage.;40:88-106

Osenberg CW. Mittelbach GG. 1989. Effects of Body Size on the Predator-Prey Interaction Between Pumpkinseed Sunfish and Gastropods.” Ecological Monographs 59(4): 405-432.

Scharf, F, Juanes F, Rountree R. 2000. Predator size-prey size relationships of marine fish predators: interspecific variation and effects of ontogeny and body size on trophic-niche breadth. Marine Ecology-progress Series - Mar Ecol-Progr Ser. 208. 229-248.

Woolgar JD, Cliff G, Nair R, Hafez H, Robbs JV. 2001. Shark attack: review of 86 consecutive cases. J. Trauma. Acute Care Surg.;50:887-891 